
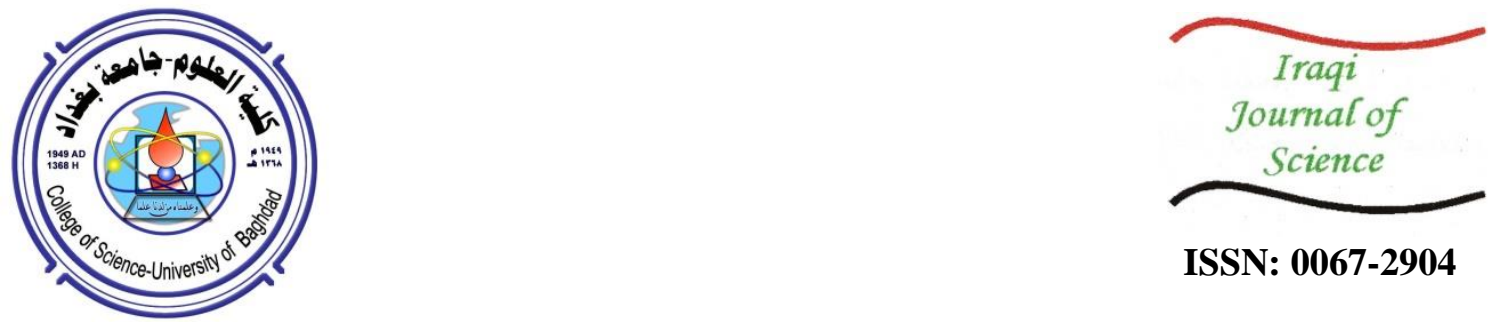

ISSN: 0067-2904

\title{
On Integrability of Christou's Sixth Order Solitary Wave Equations
}

\author{
M. Allami* \\ Department of Mathematics, College of Education, Misan University, Misan, Iraq
}

\begin{abstract}
We examine the integrability in terms of Painlevè analysis for several models of higher order nonlinear solitary wave equations which were recently derived by Christou. Our results point out that these equations do not possess Painlevè property and fail the Painlevè test for some special values of the coefficients; and that indicates a non-integrability criteria of the equations by means of the Painlevè integrability.
\end{abstract}

Keywords: Painlevè analysis, Integrability, Sixth order solitary wave equations, Painlevè property, Wiess, Tabor and Carnevale approach, Kruskal's simplification.
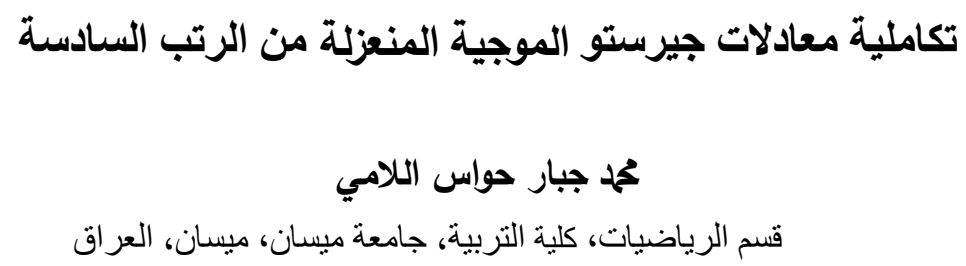

الخلاصة

بحثا بالتنصيل التكاملية من مفهوم تحليل بينليفا لمجموعة من المعادلات غير الخطية الموجية المنعزلة

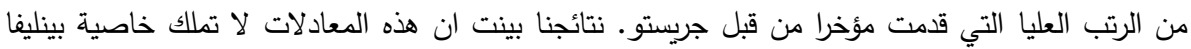

وتقشل في اختبار بينلفا لبعض القيم الخاصة للمعاملات وهذا يشير إلى معايير عدم القدرة على التكاملية

\section{Introduction}

$$
\text { للمعادلات من مفهوم تكاملية بينليفا. }
$$

A variety of new nonlinear partial differential equations were recently introduced in the work of [1] from applying a different type of techniques; The author managed to exploit fundamental physics laws, Taylor series expansion and Hirota's bilinear operator to derive some higher order solitary wave equations. The first model, the sixth order solitary wave equation using Ohm's law is given by

$$
u_{t t}-c_{1} u_{x x}-2 c_{2} u_{x}^{2}-2 c_{2} u u_{x x}+6 c_{3} u u_{x}^{2}+3 c_{3} u^{2} u_{x x}-c_{4} u_{x x x x}-c_{5} u_{x x x x x x}=0 \text {, }
$$

where $u$ is a function of $x$ and $t$, the subscripts denote to partial derivatives with respect to the independent variables, and $c_{1}=\frac{h^{2}}{C_{0} L}, c_{2}=\frac{1}{2 F_{0}}, c_{3}=\frac{1}{3 F_{0}^{2}}, c_{4}=\frac{(12 \delta+1) h^{4}}{12 C_{0} L}, c_{5}=\frac{(60 \delta+1) h^{6}}{360 C_{0} L}$ and $C_{0}=\frac{Q_{0}}{F_{0}}$. The $Q_{0}$ is the charge on the capacitor, $F_{0}$ is Faraday's constant, $L$ is the length on the $C_{0}$ capacitor, $h$ is a small parameter of the Taylor expansion and $\delta$ controls the triple interactions between sections and must be non-negative.

The travelling wave solutions for the equation (1) was obtained in [2] by using the improved generalized tanh-coth method. The second model, the sixth order solitary wave equation using Hirota's bilinear operator is given by

$$
\begin{aligned}
-u_{t t}+2 u_{x x}-15 u_{x x}^{2}-30 u_{x} u_{x x x}-15 u u_{x x x x}+90 u u_{x}^{2}+ & 45 u^{2} u_{x x}-u_{x x x x} \\
+ & u_{x x x x x x}=0 .
\end{aligned}
$$

The third model, the sixth order Sine-Gordon equation is written as

$$
\theta_{t t}-c_{0}^{2} \theta_{x x}-c_{1}^{2} \theta_{x x x x}-c_{2}^{2} \theta_{x x x x x x}=c_{3}^{2} \sin (\theta),
$$

*Email: drmjh53@gmail.com 
where $\theta$ is a function of $x$ and $t$, and $c_{j}^{2}, j=0,1,2,3$ are some physical quantities.

We shall inspect Painlevè integrability for the equations (1), (2) and (3); and the integrability means here is that the differential equation does have Painlevè property. For a given partial differential equation $\mathcal{F}\left(u, u_{z_{1}}, u_{z_{2}}, u_{z_{1} z_{1}}, \ldots\right)=0$, where $u$ is a function of $z_{1}, z_{2}, \ldots, z_{n}$, is said to have the Painlevè property if solutions are single valued about non-characteristic movable singularity manifolds, and these manifolds are determined by the condition of the form $\Phi\left(z_{1}, z_{2}, \ldots, z_{n}\right)=0$, where $\Phi$ is an analytic function. In other words, if $u\left(z_{1}, z_{2}, \ldots, z_{n}\right)$ is a solution for Partial differential equation, then it takes Laurent type expansion

$$
u\left(z_{1}, z_{2}, \ldots, z_{n}\right)=\Phi\left(z_{1}, z_{2}, \ldots, z_{n}\right)^{\lambda} \sum_{i=0}^{\infty} u_{i} \Phi\left(z_{1}, z_{2}, \ldots, z_{n}\right)^{i},
$$

where $\Phi$ and $u$ are both analytic functions, $\lambda$ is an integer number, and the number of arbitrary functions $u_{i}$ is equal to the order of the differential equation. Wiess, Tabor and Carnevale (WTC) [3] introduced an approach that one can examine singularity structure of partial differential equations directly. In addition, Wiess [4-7] investigated the Painlevè property for several partial differential equations and he showed how to construct their Bäcklund transformations and Lax pairs.

The WTC approach is basically built on three steps. Firstly, the leading order analysis, obtaining the dominant behavior of all possible singularities of the equation. Secondly, finding the resonances where arbitrary constants may occur in the Laurent expansion. Thirdly, verifying the resonance conditions in each Laurent expansion explicitly. The equation survives Painlevè test if all the three steps are satisfied. A concise review of many methods of Painlevè tests can be found in [8], and for recent applications see [9-12].

The rest of the paper is organized as follows, in section two the Painlevè analysis for the sixth order solitary wave equations using Ohm's law is considered, section three is dedicated to apply the Painlevè test for the nonlinear sixth order equation using Hirota's bilinear operator. We move to section four where the test is performed for solitary wave Sine-Gordon equation. The last section is conclusions.

\section{Painlevè analysis for the sixth order solitary wave equations using Ohm's law}

We consider the case when the coefficients of the equation (1) are taken to be $c_{1}=c_{5}=1, c_{2}=$ $\frac{1}{2}, c_{3}=\frac{1}{3}$ and $c_{4}=3$. The equation then becomes

$$
u_{t t}-u_{x x}-u_{x}^{2}-u u_{x x}+2 u u_{x}^{2}+u^{2} u_{x x}-3 u_{x x x x}-u_{x x x x x x}=0 .
$$

We search for solution of equation (5) expressed in an infinite series of Laurent type expansion

$$
u(x, t)=\Phi^{\lambda} \sum_{i=0}^{\infty} u_{i}(x, t) \Phi^{i}(x, t),
$$

in the neighborhood of a non-characteristic movable singular manifold $\Phi(x, t)=0$, and the number of arbitrary functions $u_{i}$ should be the same as the order of the equation. To determine the leading dominant behavior, let $u=\kappa \Phi^{\lambda}$, where $\kappa$ is a constant, into the equation (5) to obtain

$$
\begin{aligned}
& -\kappa \lambda\left\{3 \Phi^{\lambda-1} \Phi_{x x x x}-\Phi^{\lambda-2} \Phi_{t}^{2}-\Phi^{\lambda-1} \Phi_{t t}-18 \Phi^{\lambda-4} \Phi_{x}^{4}+30 \Phi^{\lambda-3} \Phi_{x x}^{3}-10 \Phi^{\lambda-2} \Phi_{x x x}^{2}\right. \\
& -\Phi^{\lambda-2} \Phi_{x}^{2}-9 \Phi^{\lambda-2} \Phi_{x x}^{2}+\Phi^{\lambda-1} \Phi_{x x}+\Phi^{\lambda-1} \Phi_{x x x x x}-120 \Phi^{\lambda-6} \Phi_{x}^{6}+\lambda^{5} \Phi^{\lambda-6} \Phi_{x}^{6}- \\
& \quad 15 \lambda^{5} \Phi^{\lambda-4} \Phi_{x}^{6}+85 \lambda^{3} \Phi^{\lambda-6} \Phi_{x}^{6}-225 \lambda^{2} \Phi^{\lambda-6} \Phi_{x}^{6}-3 \lambda^{3} \Phi^{\lambda-4} \Phi_{x}^{4}+274 \lambda \Phi^{\lambda-6} \Phi_{x}^{6} \\
& -18 \lambda^{2} \Phi^{\lambda-4} \Phi_{x}^{4}+33 \lambda \Phi^{\lambda-4} \Phi_{x}^{4}+15 \lambda^{2} \Phi^{\lambda-3} \Phi_{x x}^{3}+360 \Phi_{x}^{4} \Phi_{x x} \Phi^{\lambda-5}-120 \Phi^{\lambda-4} \Phi_{x x x} \Phi_{x}^{3} \\
& -270 \Phi^{\lambda-4} \Phi_{x}^{2} \Phi_{x x}^{2}-45 \lambda \Phi^{\lambda-3} \Phi_{x x}^{3}+\kappa^{2} \Phi_{x}^{2} \Phi^{3 \lambda-2}+36 \Phi^{\lambda-3} \Phi_{x}^{2} \Phi_{x x}+30 \Phi^{\lambda-3} \Phi_{x}^{2} \Phi_{x x x x} \\
& \quad+10 \lambda \Phi^{\lambda-2} \Phi_{x x x}^{2}+\lambda \Phi^{\lambda-2} \Phi_{x}^{2}+9 \lambda \Phi^{\lambda-2} \Phi_{x x}^{2}-15 \Phi^{\lambda-2} \Phi_{x x} \Phi_{x x x x}+\kappa \Phi^{2 \lambda-1} \Phi_{x x} \\
& \quad+15 \lambda^{4} \Phi^{\lambda-5} \Phi_{x}^{4} \Phi_{x x}-150 \lambda^{3} \Phi^{\lambda-5} \Phi_{x}^{4} \Phi_{x x}+20 \lambda^{3} \Phi^{\lambda-4} \Phi_{x}^{3} \Phi_{x x x}+45 \lambda^{3} \Phi^{\lambda-4} \Phi_{x}^{2} \Phi_{x x}^{2} \\
& +525 \lambda^{2} \Phi^{\lambda-5} \Phi_{x}^{4} \Phi_{x x}-120 \lambda^{2} \Phi^{\lambda-4} \Phi_{x}^{3} \Phi_{x x x}-270 \lambda^{2} \Phi^{\lambda-4} \Phi_{x}^{2} \Phi_{x x}^{2}-750 \lambda \Phi^{\lambda-5} \Phi_{x}^{4} \Phi_{x x} \\
& +220 \lambda \Phi^{\lambda-4} \Phi_{x}^{3} \Phi_{x x x}+490 \lambda \Phi^{\lambda-4} \Phi_{x}^{2} \Phi_{x x}^{2}+18 \lambda^{2} \Phi^{\lambda-3} \Phi_{x}^{2} \Phi_{x x}+15 \lambda^{2} \Phi^{\lambda-3} \Phi_{x}^{2} \Phi_{x x x x} \\
& \quad-3 \kappa^{2} \lambda \Phi^{3 \lambda-2} \Phi_{x}^{2}-54 \lambda \Phi^{\lambda-3} \Phi_{x}^{2} \Phi_{x x}-45 \lambda \Phi^{\lambda-3} \Phi_{x}^{2} \Phi_{x x x x}+120 \Phi^{\lambda-3} \Phi_{x} \Phi_{x x} \Phi_{x x x} \\
& +12 \lambda \Phi^{\lambda-2} \Phi_{x} \Phi_{x x x}+6 \lambda \Phi^{\lambda-2} \Phi_{x} \Phi_{x x x x x}+15 \lambda \Phi^{\lambda-2} \Phi_{x x} \Phi_{x x x x}+2 \kappa \lambda \Phi^{2 \lambda-2} \Phi_{x}^{2} \\
& \left.+\lambda \Phi^{\lambda-2} \Phi_{t}^{2}+60 \lambda^{2} \Phi^{\lambda-3} \Phi_{x} \Phi_{x x} \Phi_{x x x}-180 \lambda \Phi^{\lambda-3} \Phi_{x} \Phi_{x x} \Phi_{x x x}\right\}=0 .
\end{aligned}
$$

The lowest exponents of $\Phi$ are $\{2 \lambda-2,3 \lambda-2, \lambda-6\}$ and all the possible balances give the singularity orders $\lambda=-2$ and $\lambda=-4$, so the most singular terms in the equation are

and

$$
2 u u_{x}^{2}+u^{2} u_{x x}-u_{x x x x x x} \sim 0 \text {, }
$$

$$
-u_{x}^{2}-u u_{x x}-u_{x x x x x x} \sim 0 .
$$


There exist two families (7) and (8) of Painèlve expansions that needs to be discussed separately. For first family (7), inserting $u(x, t)=u_{0} \Phi^{\lambda}$ into equation (7) yields

$$
\begin{aligned}
2 u_{0}^{3} \lambda^{2} \Phi_{x}^{2} \Phi^{2 \lambda-2}-u_{0}^{3} \lambda(\lambda-1) \Phi_{x}^{2} \Phi^{2 \lambda-2}-u_{0} \lambda(\lambda-1)(\lambda-2)(\lambda-3) & (\lambda-4)(\lambda-5) \Phi_{x}^{6} \Phi^{\lambda-6} \sim 0,
\end{aligned}
$$

when $\lambda=-2$ that leads to the two branches $u_{0}=+6 \sqrt{10} \Phi_{x}^{2}$ and $u_{0}=-6 \sqrt{10} \Phi_{x}^{2}$.

In order to find the resonances, where the arbitrary constants may occur in the series, take a linear perturbation of the leading order

$$
u(x, t) \sim u_{0} \Phi^{-2}\left(1+\zeta \Phi^{r}\right),
$$

where $\zeta$ is a small parameter correction to the leading order. Substituting (9) into (7) yields

$$
\begin{aligned}
& 2\left\{4+12 \zeta \Phi^{r}-4 \mathrm{r} \zeta \Phi^{r}\right\} \Phi^{-8} u_{0}^{3} \Phi_{x}^{2}-18 \zeta \Phi^{r} u_{0}^{3} \Phi_{x}^{2} \Phi^{-8}-6 \zeta \Phi^{r} u_{0}^{3} \Phi_{x x} \Phi^{-7}+6 \zeta u_{0}^{3} \Phi_{x}^{2} \Phi^{-8} \\
& -5 r \zeta \Phi^{r} u_{0}^{3} \Phi_{x}^{2} \Phi^{-8}-2 u_{0}^{3} \Phi_{x x} \Phi^{-7}+\mathrm{r}^{2} \zeta \Phi^{r} u_{0}^{3} \Phi_{x}^{2} \Phi^{-8}-r \zeta u_{0}^{3} \Phi^{r} \Phi_{x x} \Phi^{-7}-1560 u_{0} r \zeta \\
& \Phi^{r} \Phi_{x} \Phi_{x x} \Phi_{x x x} \Phi^{-5}-540 u_{0} r^{2} \zeta \Phi^{r} \Phi_{x} \Phi_{x x} \Phi_{x x x} \Phi^{-5}+60 u_{0} r^{3} \zeta \Phi^{r} \Phi_{x} \Phi_{x x} \Phi_{x x x} \Phi^{-5} \\
& -30 u_{0} r \zeta \Phi^{r} \Phi_{x} \Phi_{x x x x x} \Phi^{-4}+1420 u_{0} r^{2} \zeta \Phi^{r} \Phi_{x}^{3} \Phi_{x x x} \Phi^{-6}+390 u_{0} r \zeta \Phi^{r} \Phi_{x}^{2} \Phi_{x x x x} \Phi^{-5} \\
& -75 u_{0} r \zeta \Phi^{r} \Phi_{x x} \Phi_{x x x x} \Phi^{-4}+15 u_{0} r^{3} \zeta \Phi^{r} \Phi_{x}^{2} \Phi_{x x x x} \Phi^{-5}+15 u_{0} r^{2} \zeta \Phi^{r} \Phi_{x x} \Phi_{x x x x} \Phi^{-4} \\
& +15660 u_{0} r \zeta \Phi^{r} \Phi_{x}^{4} \Phi_{x x} \Phi^{-7}+u_{0} r \zeta \Phi^{r} \Phi_{x x x x x x} \Phi^{-3}-1440 u_{0}\left(1+\zeta \Phi^{r}\right) \Phi_{x} \Phi_{x x} \Phi_{x x x} \Phi^{-5} \\
& +15 u_{0} r^{3} \zeta \Phi^{r} \Phi_{x x}^{3} \Phi^{-5}-135 u_{0} r^{2} \zeta \Phi^{r} \Phi_{x x}^{3} \Phi^{-5}-50 u_{0} r \zeta \Phi^{r} \Phi_{x x x}^{2} \Phi^{-4}+390 u_{0} r \zeta \Phi^{r} \\
& \Phi_{x x}^{3} \Phi^{-5}+10 u_{0} r^{2} \zeta \Phi^{r} \Phi_{x x x}^{2} \Phi^{-4}-8028 u_{0} r \zeta \Phi^{r} \Phi_{x}^{6} \Phi^{-8}-1665 u_{0} r^{3} \zeta \Phi^{r} \Phi_{x}^{6} \Phi^{-8}+ \\
& 295 u_{0} r^{4} \zeta \Phi^{r} \Phi_{x}^{6} \Phi^{-8}-27 u_{0} r^{5} \zeta \Phi^{r} \Phi_{x}^{6} \Phi^{-8}+u_{0} r^{6} \zeta \Phi^{r} \Phi_{x}^{6} \Phi^{-8}+5104 u_{0} r^{2} \zeta \Phi^{r} \Phi_{x}^{6} \Phi^{-8} \\
& +36 u_{0}\left(1+\zeta \Phi^{r}\right) \Phi_{x} \Phi_{x x x x x} \Phi^{-4}-10800 u_{0}\left(1+\zeta \Phi^{r}\right) \Phi_{x}^{4} \Phi_{x x} \Phi^{-7}+2400 u_{0}(1+ \\
& \left.\zeta \Phi^{r}\right) \Phi_{x}^{3} \Phi_{x x x} \Phi^{-6}-360 u_{0}\left(1+\zeta \Phi^{r}\right) \Phi_{x}^{2} \Phi_{x x x x} \Phi^{-5}+90 u_{0}\left(1+\zeta \Phi^{r}\right) \Phi_{x x} \Phi_{x x x x} \Phi^{-4} \\
& +5400 u_{0}\left(1+\zeta \Phi^{r}\right) \Phi_{x}^{2} \Phi_{x x}^{2} \Phi^{-6}-360 u_{0}\left(1+\zeta \Phi^{r}\right) \Phi_{x x}^{3} \Phi^{-5}+5040 u_{0}\left(1+\zeta \Phi^{r}\right) \Phi_{x}^{6} \Phi^{-8} \\
& +5040 u_{0}\left(1+\zeta \Phi^{r}\right) \Phi_{x}^{6} \Phi^{-8}++60 u_{0}\left(1+\zeta \Phi^{r}\right) \Phi_{x x x}^{2} \Phi^{-4}-2 u_{0}\left(1+\zeta \Phi^{r}\right) \Phi_{x} \Phi_{x x x x x x} \Phi^{-3} \\
& -30 u_{0} r \zeta \Phi^{r} \Phi_{x} \Phi_{x x x x x} \Phi^{-4}-75 u_{0} r \zeta \Phi^{r} \Phi_{x x} \Phi_{x x x x} \Phi^{-4}+15 u_{0} r^{3} \zeta \Phi^{r} \Phi_{x}^{2} \Phi_{x x x x} \Phi^{-5} \\
& +15 u_{0} r^{2} \zeta \Phi^{r} \Phi_{x}^{2} \Phi_{x x x x} \Phi^{-4}+15660 u_{0} r \zeta \Phi^{r} \Phi_{x}^{4} \Phi_{x x} \Phi^{-7}-300 u_{0} r^{4} \zeta \Phi^{r} \Phi_{x}^{4} \Phi_{x x} \Phi^{-7} \\
& -15 u_{0} r^{5} \zeta \Phi^{r} \Phi_{x}^{4} \Phi_{x x} \Phi^{-7}+1420 u_{0} r^{2} \zeta \Phi^{r} \Phi_{x}^{3} \Phi_{x x x} \Phi^{-6}+390 u_{0} r \zeta \Phi^{r} \Phi_{x}^{2} \Phi_{x x x x} \Phi^{-5} \\
& +3195 u_{0} r^{2} \zeta \Phi^{r} \Phi_{x}^{2} \Phi_{x x}^{2} \Phi^{-6}-820 u_{0} r^{3} \zeta \Phi^{r} \Phi_{x}^{3} \Phi_{x x x} \Phi^{-6}-3080 u_{0} r \zeta \Phi^{r} \Phi_{x}^{3} \Phi_{x x x} \Phi^{-6} \sim 0 \text {. }
\end{aligned}
$$

Now, collecting the terms that are linear in $\zeta$, and by using Kruskal's formula [13], $\Phi(x, t)=x-$ $\Theta(t)$, where $\Theta(t)$ is an arbitrary function, and setting $\Phi_{x}=1$ that gives

$$
\begin{array}{r}
\left\{\left(4(r-3)-\left(r^{2}-5 r+18\right)\right)(x-\Theta)^{r}\right\} u_{0}^{3}-\{(r-2)(r-3)(r-4)(r-5)(r-6) \\
\left.(r-7)(x-\Theta)^{r}\right\} u_{0} \sim 0,
\end{array}
$$

applying then $u_{0}=\mp 6 \sqrt{10}$ to obtain sixth degree resonances polynomial

$$
r^{6}-12 r^{5}+295 r^{4}-1665 r^{3}+4744 r^{2}-3348 r-10080=0 .
$$

Solve the algebraic equation for $r$ to gain the resonances

$$
r=-1,6,7,8, \frac{7}{2}-\frac{i \sqrt{71}}{2}, \frac{7}{2}+\frac{i \sqrt{71}}{2}, i=\sqrt{-1} .
$$

We have six resonances corresponding to sixth order partial differential equation. There exist two resonances (so-called Fuchs indices) of the first branch are non-integer numbers. The equation does not pass the Painlevè test which indicates that the equation does not have a single valued around movable singularity, but it may have a movable algebraic or logarithmic branch point. To truncate the expansion at zero order, we substitute the expression

$$
u=\frac{u_{0}}{\Phi^{2}}+\frac{u_{1}}{\Phi}+u_{2}
$$

into the equation (5) and then grouping the terms with the same power of $\Phi$ to get a set of equations, here we write down a few of these equations

$$
\begin{aligned}
& -5040 u_{0} \Phi_{x}^{6}+14 u_{0}^{3} \Phi_{x}^{2}=0 \\
& -720 u_{1} \Phi_{x}^{6}+10800 u_{0} \Phi_{x}^{4} \Phi_{x x}+4320 \Phi_{x}^{5} u_{0, x}+30 u_{0}^{2} u_{1} \Phi_{x}^{2}-2 u_{0}^{3} \Phi_{x x} \\
& \quad 720 \Phi_{x}^{5} u_{1, x}+1800 \Phi_{x}^{4} u_{0} \Phi_{x x}-360 u_{0} \Phi_{x}^{4}-1800 u_{0, x x} \Phi_{x}^{4}-7200 u_{0, x} \Phi_{x}^{3} \Phi_{x x}-2400 u_{0} \Phi_{x}^{3} \Phi_{x x x} \\
& +20 u_{0} u_{1}^{2} \Phi_{x}^{2}-5400 u_{0} \Phi_{x}^{2} \Phi_{x x}^{2}+20 u_{2} u_{0}^{2} \Phi_{x}^{2}-10 u_{0}^{2} \Phi_{x}^{2}-10 u_{0}^{2} \Phi_{x} u_{1, x}-20 u_{0} u_{1} u_{0, x} \Phi_{x}- \\
& 5 u_{1} u_{0}^{2} \Phi_{x x}+\left(u_{0}^{2} u_{0, x}\right)_{x}=0
\end{aligned}
$$

solving equation (10) for $u_{0}$ to obtain $u_{0}= \pm 6 \sqrt{10} \Phi_{x}^{2}$, plugging the value of $u_{0}$ into equation (11) and solving it for $u_{1}$ yields $u_{1}=\mp 6 \sqrt{10} \Phi_{x x}$. Using the values of $u_{0}$ and $u_{1}$ in equation (12) and solve it for $u_{2}$ to get 


$$
u_{2}=\mp \frac{20 \sqrt{10} \Phi_{x} \Phi_{x x}+3 \sqrt{10} \Phi_{x}^{2}-15 \sqrt{10} \Phi_{x x}^{2} \mp 5 \Phi_{x}^{2}}{10 \Phi_{x}^{2}} .
$$

Hence, the truncated equation of the expansion (6) at zero order is

$$
\mathrm{u}=\mp \frac{6 \sqrt{10} \Phi_{x}^{2}}{\Phi^{2}} \pm \frac{6 \sqrt{10 \Phi_{x x}}}{\Phi} \mp \frac{20 \sqrt{10} \Phi_{x} \Phi_{x x}+3 \sqrt{10} \Phi_{x}^{2}-15 \sqrt{10} \Phi_{x x}^{2} \mp 5 \Phi_{x}^{2}}{10 \Phi_{x}^{2}} .
$$

A finite number of terms here represent a local solution of the equation (5). Now, we move to deal with the second family (8). Substituting $u=u_{0} \Phi^{\lambda}$ into equation (8) to have

$$
\begin{aligned}
-u_{0}^{2} \lambda^{2} \Phi_{x}^{2} \Phi^{2 \lambda-2}-u_{0}^{2}\left(\lambda^{2}-\lambda\right) \Phi_{x}^{2} \Phi^{2 \lambda-2}-u_{0} \lambda(\lambda-1)(\lambda-2)(\lambda-3) & (\lambda-4)(\lambda-5) \Phi_{x}^{6} \Phi^{\lambda-6} \sim 0 .
\end{aligned}
$$

The dominant balancing at the singular order $\lambda=-4$ leads to the branch $u_{0}=-1680 \Phi_{x}^{4}$. For the sake of finding the resonances, taking a linear perturbation of the leading order

Substituting (13) into (8) to get

$$
u(x, t) \sim u_{0} \Phi^{-4}\left(1+\zeta \Phi^{r}\right),
$$

$$
\begin{aligned}
& -\left[\left(16-8 \zeta(r-4) \Phi^{r}+(r-4)^{2} \zeta^{2} \Phi^{2 r}\right) u_{0}^{2} \Phi_{x}^{2} \Phi^{-10}\right]-\left[\left(\zeta(r-8) \Phi^{r+1} \Phi_{x x}+(r-4)\right.\right. \\
& \left.(r-5) \zeta^{2} \Phi_{x}^{2} \Phi^{2 r}+20 \Phi_{x}^{2}+\zeta\left(r^{2}-9 r+40\right) \Phi^{r} \Phi_{x}^{2}+\Phi_{x x}\left(-4 \Phi+(r-4) \zeta^{2} \Phi^{2 r+1}\right)\right) u_{0}^{2} \\
& \left.\Phi^{-10}\right]-\left[\left(u_{0}\left(\left(\zeta(r-4) \Phi^{r+5}-4 \Phi^{5}\right) \Phi_{x x x x x x}+6\left(\zeta(r-4)(\mathrm{r}-5) \Phi^{r+4}+20 \Phi^{4}\right) \Phi_{x} \Phi_{x x x x}\right)\right.\right. \\
& +\left(15 \zeta(r-4)(r-5)(\mathrm{r}-6) \Phi_{x}^{2} \Phi^{r+3}+15 \zeta(r-4)(r-5) \Phi_{x x} \Phi^{r+4}+300 \Phi_{x x} \Phi^{4}-1800\right. \\
& \left.\Phi_{x}^{2} \Phi^{3}\right) \Phi_{x x x x}+\left(10 \zeta(r-4)(r-5) \Phi^{r+4}+200 \Phi^{4}\right) \Phi_{x x x}^{2}+20 \Phi_{x}\left(3 \Phi_{x x} \zeta(r-4)(r-5)\right. \\
& \left.(\mathrm{r}-6) \Phi^{r+3}+\zeta(r-4)(r-5)(\mathrm{r}-6)(\mathrm{r}-7) \Phi_{x}^{2} \Phi^{r+2}-360 \Phi_{x x} \Phi^{3}+840 \Phi^{2} \Phi_{x}^{2}\right) \Phi_{x} \Phi_{x x x} \\
& +15 \zeta(r-4)(r-5)(\mathrm{r}-6)(\mathrm{r}-7) \Phi_{x x}^{3} \Phi^{r+3}+45 \zeta(r-4)(r-5)(r-6)(r-7) \Phi_{x}^{2} \Phi_{x x}^{2} \Phi^{r+2} \\
& +15 \zeta(r-4)(r-5) \zeta(r-6)(r-7) \Phi_{x}^{4} \Phi_{x x} \Phi^{r+1}-1800 \Phi_{x x}^{3} \Phi^{3}+34800 \Phi_{x}^{2} \Phi_{x x}^{2} \Phi^{2}-100800 \\
& \left.\left.\left.\Phi_{x}^{4} \Phi_{x x} \Phi+\left(60480+\zeta(r-4)(r-5) \zeta(r-6)(r-7)(r-8)(r-9) \Phi^{r}\right) \Phi_{x}^{6}\right)\right) \Phi^{-10}\right] \sim 0 .
\end{aligned}
$$

Using Kruskal's formula [13], that is $\Phi(x, t)=x-\Theta(t)$, and grouping the terms linear in $\zeta$, and also setting $\Phi_{x}=1$ which comes from Kruskal's formula, to gain

$$
\left[\left(8(r-4)-\left(r^{2}-9 r+40\right)\right) u_{0}^{2}-(r-4)(r-5)(r-6)(r-7)(r-8)(r-9) u_{0}\right] \zeta \sim 0,
$$

with the benefit of $u_{0}=-1680 \Phi_{x}^{4}$, the resonances polynomial is

$$
r^{6}-39 r^{5}+625 r^{4}-5265 r^{3}+22894 r^{2}-31656 r-60480=0 .
$$

Solving the algebraic equation for $r$ to gain the Fuchs indices

$$
r=-1,8,9,12, \frac{11}{2}+\frac{1}{2} i \sqrt{159}, \frac{11}{2}-\frac{1}{2} i \sqrt{159}, i=\sqrt{-1} .
$$

Two of the resonances are non-integer numbers, thus, the equation (5) fails the test and that due to occurring of algebraic or logarithmic branch points.

\section{Painlevè analysis for the Sixth order equation using Hirota's bilinear operator}

To perform Painlevè test for equation (2), the equation is given by

$$
\begin{gathered}
-u_{t t}+2 u_{x x}-15 u_{x x}^{2}-30 u_{x} u_{x x x}-15 u u_{x x x x}+90 u u_{x}^{2}+45 u^{2} u_{x x}-u_{x x x x} \\
+u_{x x x x x x}=0 .
\end{gathered}
$$

One can deduce that the most singular terms in the equation (14) are

$$
-15 u_{x x}^{2}-30 u_{x} u_{x x x}-15 u u_{x x x x}+90 u u_{x}^{2}+45 u^{2} u_{x x}+u_{x x x x x x} \sim 0 .
$$

Substituting $u(x, t)=u_{0} \Phi^{\lambda}$ into the equation (15) yields

$$
\begin{aligned}
& -15 \lambda^{2}(\lambda-1)^{2} u_{0}^{2} \Phi_{x}^{4} \Phi^{2 \lambda-4}-30 \lambda^{2}(\lambda-1)(\lambda-2) u_{0}^{2} \Phi_{x}^{4} \Phi^{2 \lambda-4}-15 \lambda(\lambda-1)(\lambda-2)(\lambda-3) u_{0}^{2} \\
& \Phi_{x}^{4} \Phi^{2 \lambda-4}+90 \lambda^{2} u_{0}^{3} \Phi_{x}^{2} \Phi^{3 \lambda-2}+45 \lambda(\lambda-1) u_{0}^{3} \Phi_{x}^{2} \Phi^{3 \lambda-2}+\lambda(\lambda-1)(\lambda-2)(\lambda-3) \\
& (\lambda-4)(\lambda-5) u_{0} \Phi_{x}^{6} \Phi^{\lambda-6} \sim 0 .
\end{aligned}
$$

From dominant balancing, we have two branches $u_{0}=2 \Phi_{x}^{2}$ and $u_{0}=4 \Phi_{x}^{2}$ at singular order $\lambda=-2$. In order to get the resonances, Take a linear perturbation of the leading order

$$
u(x, t) \sim u_{0} \Phi^{-2}\left(1+\zeta \Phi^{r}\right),
$$

where $\zeta$ is a small correction of the leading order. Substituting (16) into equation (15) to have

$$
\begin{gathered}
-15\left[6 u_{0}\left(1+\zeta \Phi^{r}\right) \Phi_{x}^{2} \Phi^{-4}-5 u_{0} r \zeta \Phi^{r} \Phi_{x}^{2} \Phi^{-4}-2 u_{0}\left(1+\zeta \Phi^{r}\right) \Phi_{x x} \Phi^{-3}-u_{0} r^{2} \zeta \Phi^{r}\right. \\
\left.\Phi_{x}^{2} \Phi^{-4}+u_{0} r \zeta \Phi^{r} \Phi_{x x} \Phi^{-3}\right]^{2}-30\left(-2 u_{0}\left(1+\zeta \Phi^{r}\right) \Phi_{x} \Phi^{-3}+u_{0} r \zeta \Phi^{r} \Phi_{x} \Phi^{-3}\right) \\
\left(u_{0} r \zeta \Phi^{r} \Phi_{x x x} \Phi^{-3}-24 u_{0}\left(1+\zeta \Phi^{r}\right) \Phi_{x}^{3} \Phi^{-5}+18 u_{0}\left(1+\zeta \Phi^{r}\right) \Phi_{x} \Phi_{x x} \Phi^{-4}+26 u_{0}\right. \\
r \zeta \Phi^{r} \Phi_{x}^{3} \Phi^{-5}-9 u_{0} r^{2} \zeta \Phi^{r} \Phi_{x}^{3} \Phi^{-5}+u_{0} r^{3} \zeta \Phi^{r} \Phi_{x}^{3} \Phi^{-5}-2 u_{0}\left(1+\zeta \Phi^{r}\right) \Phi_{x x x} \Phi^{-3} \\
\left.-15 u_{0} r \zeta \Phi^{r} \Phi_{x} \Phi_{x x} \Phi^{-4}+3 u_{0} r^{2} \zeta \Phi^{r} \Phi_{x} \Phi_{x x} \Phi^{-4}\right)-15\left(u_{0} \Phi^{-2}\left(1+\zeta \Phi^{r}\right)\right)\left(u_{0} r \zeta\right. \\
\Phi^{r} \Phi_{x x x x} \Phi^{-3}-20 u_{0} r \zeta \Phi^{r} \Phi_{x} \Phi_{x x x} \Phi^{-4}-54 u_{0} r^{2} \zeta \Phi^{r} \Phi_{x}^{2} \Phi_{x x} \Phi^{-5}+6 u_{0} r^{3} \zeta \Phi^{r} \Phi_{x}^{2} \Phi_{x x} \\
\Phi^{-5}-15 u_{0} r \zeta \Phi^{r} \Phi_{x x}^{3} \Phi^{-4}+3 u_{0} r^{2} \zeta \Phi^{r} \Phi_{x x}^{2} \Phi^{-4}-154 u_{0} r \zeta \Phi^{r} \Phi_{x}^{4} \Phi^{-6}-120 u_{0}\left(1+\zeta \Phi^{r}\right)
\end{gathered}
$$




$$
\begin{aligned}
& \Phi_{x}^{4} \Phi^{-6}-144 u_{0}\left(1+\zeta \Phi^{r}\right) \Phi_{x}^{2} \Phi_{x x} \Phi^{-5}+18 u_{0}\left(1+\zeta \Phi^{r}\right) \Phi_{x x}^{2} \Phi^{-4}+24 u_{0}(1+ \\
& \left.\zeta \Phi^{r}\right) \Phi_{x} \Phi_{x x x} \Phi^{-4}+4 u_{0} r^{2} \zeta \Phi^{r} \Phi_{x} \Phi_{x x x} \Phi^{-4}+156 u_{0} r \zeta \Phi^{r} \Phi_{x}^{2} \Phi_{x x} \Phi^{-5}+71 u_{0} r^{2} \zeta \Phi^{r} \Phi_{x}^{4} \\
& \left.\Phi^{-6}-14 u_{0} r^{3} \zeta \Phi^{r} \Phi_{x}^{4} \Phi^{-6}+u_{0} r^{4} \zeta \Phi^{r} \Phi_{x}^{4} \Phi^{-6}-2 u_{0}\left(1+\zeta \Phi^{r}\right) \Phi_{x x x x} \Phi^{-3}\right)+ \\
& 90\left(u_{0} \Phi^{-2}\left(1+\zeta \Phi^{r}\right)\right)\left(\left[\zeta^{2}(r-2)^{2} \Phi^{2 r}-4 \zeta(r-2) \Phi^{r}+4\right] u_{0}^{2} \Phi_{x}^{2} \Phi^{-6}\right)+ \\
& 45\left(u_{0}^{2}\left(\Phi^{2 r} \zeta^{2}+2 \zeta \Phi^{r}+1\right) \Phi^{-4}\right)\left(6 u_{0}\left(1+\zeta \Phi^{r}\right) \Phi_{x}^{2} \Phi^{-4}-5 u_{0} r \zeta \Phi^{r} \Phi_{x}^{2} \Phi^{-4}-\right. \\
& \left.2 u_{0}\left(1+\zeta \Phi^{r}\right) \Phi_{x x} \Phi^{-3}-u_{0} r^{2} \zeta \Phi^{r} \Phi_{x}^{2} \Phi^{-4}+u_{0} r \zeta \Phi^{r} \Phi_{x x} \Phi^{-3}\right)+1560 u_{0} r \zeta \Phi^{r} \Phi_{x} \\
& \Phi_{x x} \Phi_{x x x} \Phi^{-5}-540 u_{0} r^{2} \zeta \Phi^{r} \Phi_{x} \Phi_{x x} \Phi_{x x x} \Phi^{-5}+60 u_{0} r^{3} \zeta \Phi^{r} \Phi_{x} \Phi_{x x} \Phi_{x x x} \Phi^{-5}{ }_{-} \\
& 30 u_{0} r \zeta \Phi^{r} \Phi_{x} \Phi_{x x x x x} \Phi^{-4}+1420 u_{0} r^{2} \zeta \Phi^{r} \Phi_{x}^{3} \Phi_{x x x} \Phi^{-6}+390 u_{0} r \zeta \Phi^{r} \Phi_{x}^{2} \Phi_{x x x x} \Phi^{-5} \\
& -75 u_{0} r \zeta \Phi^{r} \Phi_{x x} \Phi_{x x x x} \Phi^{-4}+15 u_{0} r^{3} \zeta \Phi^{r} \Phi_{x}^{2} \Phi_{x x x x} \Phi^{-5}+15 u_{0} r^{2} \zeta \Phi^{r} \Phi_{x x} \Phi_{x x x x} \Phi^{-4} \\
& +15660 u_{0} r \zeta \Phi^{r} \Phi_{x}^{4} \Phi_{x x} \Phi^{-7}+u_{0} r \zeta \Phi^{r} \Phi_{x x x x x x} \Phi^{-3}-1440 u_{0}\left(1+\zeta \Phi^{r}\right) \Phi_{x} \Phi_{x x} \Phi_{x x x} \Phi^{-5} \\
& +15 u_{0} r^{3} \zeta \Phi^{r} \Phi_{x x}^{3} \Phi^{-5}-135 u_{0} r^{2} \zeta \Phi^{r} \Phi_{x x}^{3} \Phi^{-5}-50 u_{0} r \zeta \Phi^{r} \Phi_{x x x}^{2} \Phi^{-4}+390 u_{0} r \zeta \Phi^{r} \Phi_{x x}^{3} \\
& \Phi^{-5}+10 u_{0} r^{2} \zeta \Phi^{r} \Phi_{x x x}^{2} \Phi^{-4}-8028 u_{0} r \zeta \Phi^{r} \Phi_{x}^{6} \Phi^{-8}-1665 u_{0} r^{3} \zeta \Phi^{r} \Phi_{x}^{6} \Phi^{-8}+295 u_{0} r^{4} \zeta \\
& \Phi^{r} \Phi_{x}^{6} \Phi^{-8}-27 u_{0} r^{5} \zeta \Phi^{r} \Phi_{x}^{6} \Phi^{-8}+u_{0} r^{6} \zeta \Phi^{r} \Phi_{x}^{6} \Phi^{-8}+5104 u_{0} r^{2} \zeta \Phi^{r} \Phi_{x}^{6} \Phi^{-8}+ \\
& 36 u_{0}\left(1+\zeta \Phi^{r}\right) \Phi_{x} \Phi_{x x x x x} \Phi^{-4}-10800 u_{0}\left(1+\zeta \Phi^{r}\right) \Phi_{x}^{4} \Phi_{x x} \Phi^{-7}+2400 u_{0}\left(1+\zeta \Phi^{r}\right) \\
& \Phi_{x}^{3} \Phi_{x x x} \Phi^{-6}-360 u_{0}\left(1+\zeta \Phi^{r}\right) \Phi_{x}^{2} \Phi_{x x x x} \Phi^{-5}+90 u_{0}\left(1+\zeta \Phi^{r}\right) \Phi_{x x} \Phi_{x x x x} \Phi^{-4}+ \\
& 5400 u_{0}\left(1+\zeta \Phi^{r}\right) \Phi_{x}^{2} \Phi_{x x}^{2} \Phi^{-6}-360 u_{0}\left(1+\zeta \Phi^{r}\right) \Phi_{x x}^{3} \Phi^{-5}+5040 u_{0}\left(1+\zeta \Phi^{r}\right) \Phi_{x}^{6} \Phi^{-8} \\
& +5040 u_{0}\left(1+\zeta \Phi^{r}\right) \Phi_{x}^{6} \Phi^{-8}+60 u_{0}\left(1+\zeta \Phi^{r}\right) \Phi_{x x x}^{2} \Phi^{-4}-2 u_{0}\left(1+\zeta \Phi^{r}\right) \Phi_{x} \Phi_{x x x x x x} \Phi^{-3} \\
& -30 u_{0} r \zeta \Phi^{r} \Phi_{x} \Phi_{x x x x x} \Phi^{-4}-75 u_{0} r \zeta \Phi^{r} \Phi_{x x} \Phi_{x x x x} \Phi^{-4}+15 u_{0} r^{3} \zeta \Phi^{r} \Phi_{x}^{2} \Phi_{x x x x} \Phi^{-5}+ \\
& 15 u_{0} r^{2} \zeta \Phi^{r} \Phi_{x}^{2} \Phi_{x x x x} \Phi^{-4}+15660 u_{0} r \zeta \Phi^{r} \Phi_{x}^{4} \Phi_{x x} \Phi^{-7}-300 u_{0} r^{4} \zeta \Phi^{r} \Phi_{x}^{4} \Phi_{x x} \Phi^{-7}- \\
& 15 u_{0} r^{5} \zeta \Phi^{r} \Phi_{x}^{4} \Phi_{x x} \Phi^{-7}+1420 u_{0} r^{2} \zeta \Phi^{r} \Phi_{x}^{3} \Phi_{x x x} \Phi^{-6}+390 u_{0} r \zeta \Phi^{r} \Phi_{x}^{2} \Phi_{x x x x} \Phi^{-5} \\
& +3195 u_{0} r^{2} \zeta \Phi^{r} \Phi_{x}^{2} \Phi_{x x}^{2} \Phi^{-6}-820 u_{0} r^{3} \zeta \Phi^{r} \Phi_{x}^{3} \Phi_{x x x} \Phi^{-6}-3080 u_{0} r \zeta \Phi^{r} \Phi_{x}^{3} \Phi_{x x x} \Phi^{-6} \sim 0 \text {. }
\end{aligned}
$$

Using Kruskal's formula [13], $\Phi(x, t)=x-\Theta(t)$, and keeping only the coefficients linear in $\zeta$, with $\Phi_{x}=1$, to obtain

$-15\left[12(r-2)(r-3) u_{0}^{2}\right]-30\left[-2(r-2)\left(r^{2}-7 r+24\right) u_{0}^{2}\right]-15\left[r^{4}-14 r^{3}+71 r^{2}-154 r+\right.$ $\left.\left.240) u_{0}^{2}\right]+90\left[-4(r-3) u_{0}^{3}\right]+45\left[r^{2}-15 r+18\right) u_{0}^{3}\right]+[(r-2)(r-3)(r-4)(r-5)(r-$ 6) $\left.(r-7) u_{0}\right]=0$

applying $u_{0}=2 \Phi_{x}^{2}$ to have the resonances polynomial

$$
2 r^{6}-54 r^{5}+530 r^{4}-2250 r^{3}+3428 r^{2}+1224 r-5040=0,
$$

solving the last equation for $r$ to gain Fuchs indices $r=-1,2,3,6,7,10$.

Also, for the other branch, applying $u_{0}=4 \Phi_{x}^{2}$ gives

$$
4 r^{6}-108 r^{5}+940 r^{4}-2340 r^{3}-5264 r^{2}+18288 r+20160=0,
$$

solving the last equation to gain Fuchs indices $r=-2,-1,5,6,7,12$. The resonance $r=-1$, so called universal resonance, is corresponding to the arbitrary manifold $\Phi$. For more details about negative resonances we refer the reader to $[14,15]$. We consider only the principle branch, when $u_{0}=2 \Phi_{x}^{2}$, to verify the compatibility conditions. Substituting $u(x, t)=\sum_{i=0}^{10} u_{i} \Phi^{i-2}$ into equation (15) and collecting the coefficients of $\Phi^{i}$. Here we write down a few of these equations

$$
\begin{aligned}
& \quad 5040 \Phi_{x}^{6} u_{0}-3780 \Phi_{x}^{4} u_{0}^{2}+630 \Phi_{x}^{2} u_{0}^{3}=0 \\
& \quad 720 \Phi_{x}^{6} u_{1}-4320 \Phi_{x}^{5} u_{0, x}-3600 \Phi_{x}^{4} u_{0} u_{1}-10800 \Phi_{x x} \Phi_{x}^{4} u_{0}+3960 \Phi_{x}^{3} u_{0, x} u_{0}+1350 \Phi_{x}^{2} u_{0}^{2} u_{1} \\
& \quad+3600 \Phi_{x x} \Phi_{x}^{2} u_{0}^{2}-540 \Phi_{x} u_{0, x} u_{0}^{2}-90 \Phi_{x x} u_{0}^{3}=0 \\
& -720 \Phi_{x}^{5} u_{1, x}-600 \Phi_{x}^{4} u_{1}^{2}-1800 \Phi_{x x} \Phi_{x}^{4} u_{1}-1800 \Phi_{x}^{4} u_{0} u_{2}-120 \Phi_{x}^{4} u_{0}+1800 u_{0, x x} \Phi_{x}^{4} \\
& \quad+2400 \Phi_{x}^{3} u_{0, x} u_{1}+7200 \Phi_{x x} \Phi_{x}^{3} u_{0, x}+2400 \Phi_{x x x} \Phi_{x}^{3} u_{0}+1800 \Phi_{x}^{3} u_{1, x} u_{0}+900 \Phi_{x}^{2} u_{0} u_{1}^{2} \\
& +3900 \Phi_{x x} \Phi_{x}^{2} u_{0} u_{1}+900 \Phi_{x}^{2} u_{2} u_{0}^{2}+5400 \Phi_{x x}^{2} \Phi_{x}^{2} u_{0}-780 \Phi_{x}^{2} u_{0, x}^{2}-1080 \Phi_{x}^{2} u_{0, x x} u_{0} \\
& -900 \Phi_{x} u_{0, x} u_{0} u_{1}-2220 \Phi_{x} \Phi_{x x} u_{0, x} u_{0}-480 \Phi_{x} \Phi_{x x x} u_{0}^{2}-450 \Phi_{x} u_{1, x} u_{0}^{2}-225 \Phi_{x x} u_{0}^{2} u_{1} \\
& \quad-330 \Phi_{x x}^{2} u_{0}^{2}+90 u_{0, x}^{2} u_{0}+45 u_{0, x x} u_{0}^{2}=0 \\
& \quad 180 \Phi_{x x x} u_{0, x} u_{0}+840 \Phi_{x}^{3} u_{1} u_{1, x}+480 \Phi_{x x x} \Phi_{x}^{3} u_{1}+720 \Phi_{x}^{3} u_{0} u_{2, x}+1440 \Phi_{x}^{3} u_{0, x} u_{2} \\
& \quad+540 \Phi_{x}^{2} u_{3} u_{0}^{2}-2160 \Phi_{x} \Phi_{x x}^{2} u_{0, x}-420 \Phi_{x x}^{2} u_{0} u_{1}-2160 \Phi_{x x} \Phi_{x}^{2} u_{0, x x}+144 \Phi_{x x} \Phi_{x}^{2} u_{0} \\
& -180 \Phi_{x x} u_{0} u_{1}^{2}-180 \Phi_{x x} u_{2} u_{0}^{2}-540 \Phi_{x}^{2} u_{0} u_{1, x x}-780 \Phi_{x}^{2} u_{1} u_{0, x x}-960 \Phi_{x}^{2} u_{0, x} u_{1, x} \\
& -360 \Phi_{x x x x} \Phi_{x}^{2} u_{0}-1440 \Phi_{x x x} \Phi_{x}^{2} u_{0, x}-360 \Phi_{x} u_{0, x} u_{1}^{2}-360 \Phi_{x} u_{2, x} u_{0}^{2}+240 \Phi_{x x} u_{0, x x} u_{0} \\
& +300 \Phi_{x} u_{0, x} u_{0, x x}+180 \Phi_{x} u_{0} u_{0, x x x}+90 u_{0, x x} u_{0} u_{1}+180 u_{0} u_{0, x} u_{1, x}-1080 \Phi_{x}^{4} u_{0} u_{3} \\
& -360 \Phi_{x}^{4} u_{2} u_{1}+1080 \Phi_{x}^{2} \Phi_{x x}^{2} u_{1}+1440 \Phi_{x x} \Phi_{x}^{3} u_{1, x}+780 \Phi_{x x} \Phi_{x}^{2} u_{1}^{2}+180 \Phi_{x}^{2} u_{1}^{3} \\
& -360 \Phi_{x x}^{3} u_{0}+96 \Phi_{x}^{3} u_{0, x}-480 \Phi_{x}^{3} u_{0, x x x}+180 \Phi_{x x} u_{0, x}^{2}+45 u_{1, x x} u_{0}^{2}+90 u_{0, x}^{2} u_{1}
\end{aligned}
$$




$$
\begin{gathered}
+30 \Phi_{x x x x} u_{0}^{2}+360 \Phi_{x}^{4} u_{1, x x}-24 \Phi_{x}^{2} u_{1}+2160 \Phi_{x x} \Phi_{x}^{2} u_{0} u_{2}+1080 \Phi_{x}^{2} u_{0} u_{1} u_{2} \\
-1560 \Phi_{x x} \Phi_{x} u_{1} u_{0, x}-1200 \Phi_{x x} \Phi_{x} u_{1, x} u_{0}-1440 \Phi_{x x x} \Phi_{x x} \Phi_{x} u_{0}-720 \Phi_{x} u_{0} u_{1, x} u_{1} \\
-600 \Phi_{x x x} \Phi_{x} u_{1} u_{0}-720 \Phi_{x} u_{2} u_{0, x} u_{0}=0
\end{gathered}
$$

where $u_{j, x}=\frac{\partial u_{j}}{\partial x}, u_{j, x x}=\frac{\partial^{2} u_{j}}{\partial x^{2}}, u_{j, x x x}=\frac{\partial^{3} u_{j}}{\partial x^{3}} j=0,1,2,3$. Solving equation (17) to obtain, by using Kruskal's simplification [13], $\Phi(x, t)=x-\Theta(t)$, the following branches $u_{0}=2 \Phi_{x}^{2} \quad$ and $u_{0}=4 \Phi_{x}^{2}$. From equation (18) we have

$$
-1080 u_{1} \Phi_{x}^{6}-2160 \Phi_{x}^{6} \Phi_{x x}=0 \text { and } u_{1}=-2 \Phi_{x x} \text { and so } u_{1}=0 .
$$

Also, from equation (19) one can get $-240 \Phi_{x}^{6}=0$, and since $\Phi_{x}=1$ that leads to $-240=0$ which is inconsistent. Therefore, the compatibility condition is not satisfied and the equation (14) fails the Painlevè test.

\section{Painlevè analysis for sixth order Sine-Gordon equation}

We discuss the case when the coefficients of the equation (3) are taken as $c_{0}^{2}=c_{1}^{2}=c_{2}^{2}=c_{3}^{2}=1$. The equation then is given by

$$
\theta_{t t}-\theta_{x x}-\theta_{x x x x}-\theta_{x x x x x x}=\sin (\theta)
$$

adapting the transformation $u=\exp (i \theta), i=\sqrt{-1}$, with the benefit of the relation $\sin (\theta)=$ $\frac{\exp (i \theta)-\exp (-i \theta)}{2 i}$, to get the equation

$$
\begin{aligned}
& u^{7}-2 u_{2 t} u^{5}+2 u_{t}^{2} u^{4}-240 u_{x}^{6}-12 u_{x}^{4} u^{2}+720 u_{x}^{4} u_{x x} u-240 u_{x x x} u_{x}^{3} u^{2}-2 u_{x}^{2} u^{4} \\
& +60 u_{x x x x} u_{x}^{2} u^{3}+24 u_{x x} u_{x}^{2} u^{3}-540 u_{x x}^{2} u_{x}^{2} u^{2}-8 u_{x x x} u_{x} u^{4}-12 u_{x x x x} u_{x} u^{4} \\
& +240 u_{x x x} u_{x} u_{x x} u^{3}+2 u_{x x x x} u^{5}+2 u_{x x} u^{5}+2 u_{x x x x x x} u^{5}-u^{5}-30 u_{x x x x} u_{x x} u^{4} \\
& -6 u_{x x}^{2} u^{4}-20 u_{x x x}^{2} u^{4}+60 u_{x x}^{3} u^{3}=0 .
\end{aligned}
$$

To determine the dominant behavior, plug $u=u_{0} \Phi^{\lambda}$ into the equation (22) to obtain

$$
\begin{aligned}
& \quad-u_{0}^{5}\left[-u_{0}^{2} \Phi^{7 \lambda}+2 u_{0} \kappa \lambda \Phi^{6 \lambda-1} \Phi_{t t}-2 u_{0} \lambda \Phi^{6 \lambda-2} \Phi_{t}^{2}+30 u_{0} \lambda \Phi^{6 \lambda-2} \Phi_{x x} \Phi_{x x x x}\right. \\
& +12 u_{0} \lambda \Phi^{6 \lambda-2} \Phi_{x} \Phi_{x x x x x}-60 u_{0} \lambda \Phi^{6 \lambda-3} \Phi_{x x x x} \Phi_{x}^{2}+\Phi^{5 \lambda}+240 u_{0} \lambda \Phi^{6 \lambda-4} \Phi_{x x x} \Phi_{x}^{3} \\
& -720 u_{0} \lambda \Phi^{6 \lambda-5} \Phi_{x x} \Phi_{x}^{4}+540 u_{0} \lambda \Phi^{6 \lambda-4} \Phi_{x}^{2} \Phi_{x x}^{2}+8 u_{0} \lambda \Phi_{x} \Phi_{x x x}-24 u_{0} \lambda \Phi^{6 \lambda-3} \Phi_{x x} \Phi_{x}^{2} \\
& \quad+240 u_{0} \lambda \Phi^{6 \lambda-6} \Phi_{x}^{6}-60 u_{0} \lambda \Phi^{6 \lambda-3} \Phi_{x x}^{3}+20 u_{0} \lambda \Phi^{6 \lambda-2} \Phi_{x x x}^{2}+6 u_{0} \lambda \Phi^{6 \lambda-2} \Phi_{x x}^{2} \\
& \quad+12 u_{0} \lambda \Phi^{6 \lambda-4} \Phi_{x}^{4} \Phi^{\lambda-2}-240 u_{0} \lambda \Phi^{6 \lambda-3} \Phi_{x} \Phi_{x x} \Phi_{x x x}-2 u_{0} \lambda \Phi^{6 \lambda-1} \Phi_{x x x x x x} \\
& \left.-2 u_{0} \lambda \Phi^{6 \lambda-1} \Phi_{x x}+2 u_{0} \lambda \Phi^{6 \lambda-2} \Phi_{x}^{2}-2 u_{0} \lambda \Phi^{6 \lambda-1} \Phi_{x x x x}\right] \sim 0,
\end{aligned}
$$

From the last equation, one can get the branch $u_{0}=-1440 \Phi_{x}^{6}$ at singular order $\lambda=-6$.

To detect the resonances where arbitrary constants may occur in the equation, take a linear perturbation of the leading order, that is

inserting (23) into equation (22) to have

$$
u(x, t) \sim u_{0} \Phi^{-6}\left(1+\zeta \Phi^{r}\right)
$$

$$
\left[u_{0} \Phi^{-6}\left(1+\zeta \Phi^{r}\right)\right]^{7}-240\left[u_{0} \Phi_{x}\left(-6+\zeta(\mathrm{r}-6) \Phi^{r}\right) \Phi^{-7}\right]^{6}-12\left[u_{0} \Phi_{x}(-6+\zeta(\mathrm{r}-\right.
$$

6) $\left.\Phi^{r} \Phi^{-7}\right]^{4}\left[u_{0}\left(1+\zeta \Phi^{r}\right) \Phi^{-6}\right]^{2}+720\left[u_{0} \Phi_{x}\left(-6+\zeta(\mathrm{r}-6) \Phi^{r}\right) \Phi^{-7}\right]^{4}[(42+\zeta(\mathrm{r}-6)(\mathrm{r}-$ 7) $\left.\left.\Phi_{x}^{2} \Phi^{r}+\Phi_{x x}\left(-6 \Phi+(\mathrm{r}-6) \zeta \Phi^{r+1}\right)\right) u_{0} \Phi^{-8}\right]\left[u_{0}\left(1+\zeta \Phi^{r}\right) \Phi^{-6}\right]-240\left[u_{0}\left(\left(\zeta \Phi^{r+2}(r-6)-\right.\right.\right.$ $\left.6 \Phi^{2}\right) \Phi_{x x x}+\left(3 \Phi_{x x} \zeta \Phi^{r+1}(r-6)(r-7)+126 \Phi_{x x} \Phi+\Phi_{x}^{2}\left(-336+\zeta \Phi^{r}(r-6)(r-7)(r-\right.\right.$ 8))) $\left.\left.\Phi_{x}\right) \Phi^{-9}\right]\left[u_{0} \Phi_{x}\left(-6+\zeta(\mathrm{r}-6) \Phi^{r}\right) \Phi^{-7}\right]^{3}\left[u_{0}\left(1+\zeta \Phi^{r}\right) \Phi^{-6}\right]^{2} \quad-2\left[u_{0} \Phi_{x}(-6+\zeta(\mathrm{r}-\right.$ 6) $\left.\left.\Phi^{r}\right) \Phi^{-7}\right]^{2}\left[u_{0} \Phi^{-6}\left(1+\zeta \Phi^{r}\right)\right]^{4}+60\left[\left(u_{0}\left(\left(\zeta(\mathrm{r}-6) \Phi^{r+3}-6 \Phi^{3}\right) \Phi_{x x x x}+4 \Phi_{x}\left(\zeta \Phi^{r+2}(r-\right.\right.\right.\right.$ 6) $\left.(r-7)+42 \Phi^{2}\right) \Phi_{x x x}+3 \Phi_{x x}^{2} \zeta \Phi^{r+2}(r-6)(r-7)+6 \Phi_{x x} \Phi_{x}^{2} \zeta \Phi^{r+1}(r-6)(r-7)(r-8)+$ $\left.\left.\left.126 \Phi_{x x}^{2} \Phi^{2}-2016 \Phi_{x}^{2} \Phi_{x x} \Phi+\Phi_{x}^{4}\left(3024+\zeta \Phi^{r}(r-6)(r-7)(r-8)(-9)\right)\right)\right) \Phi^{-10}\right]\left[u_{0} \Phi_{x}(-6+\right.$ $\left.\left.\zeta(\mathrm{r}-6) \Phi^{r}\right) \Phi^{-7}\right]^{2}\left[u_{0} \Phi^{-6}\left(1+\zeta \Phi^{r}\right)\right]^{3}+24\left[\left(42+\zeta(\mathrm{r}-6)(\mathrm{r}-7) \Phi_{x}^{2} \Phi^{r}+\Phi_{x x}(-6 \Phi+(\mathrm{r}-\right.\right.$ 6) $\left.\left.\left.\zeta \Phi^{r+1}\right)\right) u_{0} \Phi^{-8}\right]\left[u_{0} \Phi_{x}\left(-6+\zeta(\mathrm{r}-6) \Phi^{r}\right) \Phi^{-7}\right]^{2}\left[u_{0} \Phi^{-6}\left(1+\zeta \Phi^{r}\right)\right]^{3}-540[(42+\zeta(\mathrm{r}-6)(\mathrm{r}-$ 7) $\left.\left.\Phi_{x}^{2} \Phi^{r}+\Phi_{x x}\left(-6 \Phi+(\mathrm{r}-6) \zeta \Phi^{r+1}\right)\right) u_{0} \Phi^{-8}\right]^{2}\left[u_{0} \Phi_{x}\left(-6+\zeta(\mathrm{r}-6) \Phi^{r}\right) \Phi^{-7}\right]^{2}\left[u_{0} \Phi^{-6}(1+\right.$ $\left.\left.\zeta \Phi^{r}\right)\right]^{2}-8\left[u_{0}\left(\left(\zeta \Phi^{r+2}(r-6)-6 \Phi^{2}\right) \Phi_{x x x}+\left(3 \Phi_{x x} \zeta \Phi^{r+1}(r-6)(r-7)+126 \Phi_{x x} \Phi+\right.\right.\right.$ $\left.\left.\left.\Phi_{x}^{2}\left(-336+\zeta \Phi^{r}(r-6)(r-7)(r-8)\right)\right) \Phi_{x}\right) \Phi^{-9}\right]\left[u_{0} \Phi_{x}\left(-6+\zeta(\mathrm{r}-6) \Phi^{r}\right) \Phi^{-7}\right]\left[u_{0} \Phi^{-6}(1+\right.$ $\left.\left.\zeta \Phi^{r}\right)\right]^{4}-12\left[\left(\left(\left(r \zeta \Phi^{r+4}-6 \Phi^{4}\left(1+\zeta \Phi^{r}\right)\right) \Phi_{x x x x x}\right)+5\left(\mathrm{r} \zeta(r-13) \Phi^{r+3}+42 \Phi^{3}(1+\right.\right.\right.$ $\left.\left.\zeta \Phi^{r}\right)\right) \Phi_{x} \Phi_{x x x x}+\left(10 \mathrm{r} \zeta \Phi_{x}^{2}\left(r^{2}-21 \mathrm{r}+146\right) \Phi^{r+2}+10 \mathrm{r} \Phi_{x x} \zeta(\mathrm{r}-13) \Phi^{r+3}+420 \Phi^{2}(1+\right.$ $\left.\left.\zeta \Phi^{r}\right)\left(\Phi_{x x} \Phi-8 \Phi_{x}^{2}\right)\right) \Phi_{x x x}+\Phi_{x}\left(15 \mathrm{r} \Phi_{x x}^{2} \zeta\left(r^{2}-21 \mathrm{r}+146\right) \Phi^{r+2}+10 \mathrm{r} \zeta \Phi_{x}^{2} \Phi_{x x}(\mathrm{r}-15)\left(r^{2}-\right.\right.$ $15 \mathrm{r}+110) \Phi^{r+1}-5040 \Phi^{2}\left(1+\zeta \Phi^{r}\right) \Phi_{x x}^{2}+30240 \Phi \Phi_{x}^{2}\left(1+\zeta \Phi^{r}\right) \Phi_{x x}+\Phi_{x}^{4}\left(-30240+\zeta \Phi^{r}(r-\right.$ 6) $\left.\left.(r-7)(r-8)(r-9)(r-10))) u_{0}\right) \Phi^{-11}\right]\left[u_{0} \Phi_{x}\left(-6+\zeta(r-6) \Phi^{r}\right) \Phi^{-7}\right]\left[u_{0} \Phi^{-6}\left(1+\zeta \Phi^{r}\right)\right]^{4}+$ $240\left[\left(42+\zeta(\mathrm{r}-6)(\mathrm{r}-7) \Phi_{x}^{2} \Phi^{r}+\Phi_{x x}\left(-6 \Phi+(\mathrm{r}-6) \zeta \Phi^{r+1}\right)\right) u_{0} \Phi^{-8}\right]\left[u_{0} \Phi_{x}(-6+\right.$ 
$\left.\left.\zeta(\mathrm{r}-6) \Phi^{r}\right) \Phi^{-7}\right]\left[u_{0} \Phi^{-6}\left(1+\zeta \Phi^{r}\right)\right]^{3}\left[u_{0}\left(\left(\zeta \Phi^{r+2}(r-6)-6 \Phi^{2}\right) \Phi_{x x x}+\left(3 \Phi_{x x} \zeta \Phi^{r+1}(r-6)(r-\right.\right.\right.$ $\left.\left.\left.7)+126 \Phi_{x x} \Phi+\Phi_{x}^{2}\left(-336+\zeta \Phi^{r}(r-6)(r-7)(r-8)\right)\right) \Phi_{x}\right) \Phi^{-9}\right]\left[u_{0} \Phi_{x}(-6+\zeta(\mathrm{r}-\right.$ 6) $\left.\left.\Phi^{r}\right) \Phi^{-7}\right]+2\left[\left(u_{0}\left(\left(\zeta(\mathrm{r}-6) \Phi^{r+3}-6 \Phi^{3}\right) \Phi_{x x x x}+4 \Phi_{x}\left(\zeta \Phi^{r+2}(r-6)(r-7)+42 \Phi^{2}\right) \Phi_{x x x}+\right.\right.\right.$ $3 \Phi_{x x}^{2} \zeta \Phi^{r+2}(r-6)(r-7)+6 \Phi_{x x} \Phi_{x}^{2} \zeta \Phi^{r+1}(r-6)(r-7)(r-8)+126 \Phi_{x x}^{2} \Phi^{2}-$ $\left.\left.\left.2016 \Phi_{x}^{2} \Phi_{x x} \Phi+\Phi_{x}^{4}\left(3024+\zeta \Phi^{r}(r-6)(r-7)(r-8)(-9)\right)\right)\right) \Phi^{-10}\right]\left[u_{0} \Phi^{-6}\left(1+\zeta \Phi^{r}\right)\right]^{5}+$ $2\left[\left(42+\zeta(\mathrm{r}-6)(\mathrm{r}-7) \Phi_{x}^{2} \Phi^{r}+\Phi_{x x}\left(-6 \Phi+(\mathrm{r}-6) \zeta \Phi^{r+1}\right)\right) u_{0} \Phi^{-8}\right]\left[u_{0} \Phi^{-6}\left(1+\zeta \Phi^{r}\right)\right]^{5}+$ $2\left[\left(u_{0}\left(\left(r \zeta \Phi^{r+5}-6\left(1+\zeta \Phi^{r}\right) \Phi^{5}\right)\right) \Phi_{x x x x x x}+6 \Phi_{x}\left(r \zeta(r-13) \Phi^{r+4}+42\left(1+\zeta \Phi^{r}\right) \Phi^{4}\right)\right) \Phi_{x x x x x}+\right.$ $\left(15 r \zeta \Phi_{x}^{2}\left(r^{2}-21 \mathrm{r}+146\right) \Phi^{r+3}+15 r \Phi_{x x} \zeta(r-13) \Phi^{r+4}+630\left(1+\zeta \Phi^{r}\right)\left(\Phi_{x x} \Phi-\right.\right.$ $\left.\left.8 \Phi_{x}^{2}\right)\right) \Phi_{x x x x}+\left(10 r\left(1+\zeta \Phi^{r}\right) \Phi^{4}\right) \Phi_{x x x}^{2}+20 \Phi_{x}\left(3 r \Phi_{x x} \zeta\left(r^{2}-21 r+146\right) \Phi^{r+3} \quad r \zeta \Phi_{x}^{2}(r-\right.$ $\left.15)\left(r^{2}-15 \mathrm{r}+110\right) \Phi^{r+2}-1008 \Phi^{2}\left(1+\zeta \Phi^{r}\right)\left(\Phi_{x x} \Phi-83\right)\right) \Phi_{x x x}+15 r \Phi_{x x}^{3} \quad \zeta\left(r^{2}-21 \mathrm{r}+\right.$ 146) $\Phi^{r+3}+45 r \Phi_{x x}^{2} \Phi_{x}^{2} \zeta(r-15)\left(r^{2}-15 r+110\right) \Phi^{r+2}+15 r \Phi_{x x} \Phi_{x}^{4} \zeta \quad\left(r^{4}-40 r^{3}+635 r^{2}-\right.$ $\left.5000 r+19524) \Phi^{r+1}-5040\left(1+\zeta \Phi^{r}\right) \Phi^{3}\right) \Phi_{x x}^{3}+136080 \Phi^{2} \Phi_{x}^{2}$

$\left(1+\zeta \Phi^{r}\right) \Phi_{x x}^{2}-453600 \Phi \Phi_{x}^{4}\left(1+\zeta \Phi^{r}\right) \Phi_{x x}+(332640+\zeta(r-6)(r-7)(r-8)(r-9)(r-$ 10) $\left.\left.\left.\left.(r-11) \Phi^{r}\right) \Phi_{x}^{6}\right)\right) \Phi^{-12}\right]-2\left[\left(\left(\zeta \Phi^{r+2}(r-6)-6 \Phi^{2}\right) \Phi_{x x}+\Phi_{x}\left(3 \Phi_{x x} \zeta(r-6)(r-7) \Phi^{r+1}+\right.\right.\right.$ $\left.\left.\left.126 \Phi \Phi_{x x}+\Phi_{x}^{2}\left(-336+\zeta(r-6)(r-7)(r-8) \Phi^{r}\right)\right)\right) u_{0} \Phi^{-9}\right]\left[u_{0} \Phi^{-6}\left(1+\zeta \Phi^{r}\right)\right]^{5}-$ $30\left[\left(u_{0}\left(\left(\zeta(\mathrm{r}-6) \Phi^{r+3}-6 \Phi^{3}\right) \Phi_{x x x x}+4 \Phi_{x}\left(\zeta \Phi^{r+2}(r-6)(r-7)+42 \Phi^{2}\right) \Phi_{x x x}+\right.\right.\right.$ $3 \Phi_{x x}^{2} \zeta \Phi^{r+2}(r-6)(r-7)+6 \Phi_{x x} \Phi_{x}^{2} \zeta \Phi^{r+1}(r-6)(r-7)(r-8)+126 \Phi_{x x}^{2} \Phi^{2}-$ $\left.\left.\left.2016 \Phi_{x}^{2} \Phi_{x x} \Phi+\Phi_{x}^{4}\left(3024+\zeta \Phi^{r}(r-6)(r-7)(r-8)(r-9)\right)\right)\right) \Phi^{-10}\right]\left[u_{0} \Phi^{-6}\left(1+\zeta \Phi^{r}\right)\right]^{4}$ $\left[\left(42+\zeta(\mathrm{r}-6)(\mathrm{r}-7) \Phi_{x}^{2} \Phi^{r}+\Phi_{x x}\left(-6 \Phi+(\mathrm{r}-6) \zeta \Phi^{r+1}\right)\right) u_{0} \Phi^{-8}\right]-6[(42+\zeta(\mathrm{r}-6)(\mathrm{r}-$ 7) $\left.\left.\Phi_{x}^{2} \Phi^{r}+\Phi_{x x}\left(-6 \Phi+(\mathrm{r}-6) \zeta \Phi^{r+1}\right)\right) u_{0} \Phi^{-8}\right]^{2}\left[u_{0} \Phi^{-6}\left(1+\zeta \Phi^{r}\right)\right]^{4}-20\left[u_{0}\left(\left(\zeta \Phi^{r+2}(r-6)-\right.\right.\right.$ $\left.6 \Phi^{2}\right) \Phi_{x x x}+\left(3 \Phi_{x x} \zeta \Phi^{r+1}(r-6)(r-7)+126 \Phi_{x x} \Phi+\Phi_{x}^{2}\left(-336+\zeta \Phi^{r}(r-6)(r-7)(r-\right.\right.$ 8))) $\left.\left.\Phi_{x}\right) \Phi^{-9}\right]^{2}\left[u_{0} \Phi^{-6}\left(1+\zeta \Phi^{r}\right)\right]^{4}+2\left[\left(\left(42+\zeta \Phi^{r}(r-6)(r-7) \Phi_{x}^{2}+\Phi_{x x}(-6 \Phi+(r-\right.\right.\right.$ 6) $\left.\left.\zeta \Phi^{r+1}\right) u_{0} \Phi^{-8}\right]\left[u_{0} \Phi^{-6}\left(1+\zeta \Phi^{r}\right)\right]^{4}+60\left[\left(42+\zeta(\mathrm{r}-6)(\mathrm{r}-7) \Phi_{x}^{2} \Phi^{r}+\Phi_{x x}(-6 \Phi+(\mathrm{r}-\right.\right.$ 6) $\left.\left.\left.\zeta \Phi^{r+1}\right)\right) u_{0} \Phi^{-8}\right]^{3}\left[u_{0} \Phi^{-6}\left(1+\zeta \Phi^{r}\right)\right]^{3} \sim 0$.

Taking into account Kruskal's formula [13], $\Phi(x, t)=x-\Theta(t)$, where $\Theta(t)$ is an arbitrary function, and $u_{0}=-1440 \Phi_{x}^{6}$, and $\Phi_{x}=1$ to have sixth degree resonances polynomial

$$
r^{6}-15 r^{5}+85 r^{4}-225 r^{3}+274 r^{2}-120 r-720=0 .
$$

Solving the last equation for $r$ to get

$$
r=-1,6, \frac{5}{2}-\frac{1}{2} \sqrt{-7-8 i \sqrt{14}}, \frac{5}{2}+\frac{1}{2} \sqrt{-7-8 i \sqrt{14}}, \frac{5}{2}-\frac{1}{2} \sqrt{-7+8 i \sqrt{14}}, \frac{5}{2}+\frac{1}{2} \sqrt{-7+8 i \sqrt{14}}
$$

Obviously, the branch possesses non-integer resonances and the equation does not pass the Painlevé test.

\section{Conclusion}

After all what we have discussed in the current work, it seems to be that all examined equations do not survive the Painlevè test, and therefore they are not integrable, in the Painlevè sense, for some special values of the coefficients. The Painlevé analysis, in fact, gives us an idea about the nature of solutions of the equations. The bad positions of resonances provide an evidence on occurring of algebraic or logarithmic branch points in their solutions, and the inconsistent of the compatibility conditions making the equations do not pass the test. The test for other special values of the coefficients or even the more general cases of the equations that needs to be considered in the future work.

\section{References}

1. Christou MA. 2017. Sixth order solitary wave equations. Wave Motion, (71):18-24.

2. Torvattanabun M, Simmapim J, Saennuad D, Somaumchan T. 2017. The improved generalized tanh-coth method applied to sixth-order solitary wave equation. Journal of Mathematics, 2017, https://doi.org/10.1155/2017/8751097.

3. Weiss J, Tabor M, Carnevale M. 1983. The Painlevè property for partial differential equations, Journal of Mathematical Physics, 24(3): 522-6.

4. Weiss J. 1983. The Painlevé property for partial differential equations. II: Bäcklund transformation, Lax pairs, and the Schwarzian derivative, Journal of Mathematical Physics, 24(6): 1405-13.

5. Weiss J. 1984. On classes of integrable systems and the Painlevé property, Journal of Mathematical Physics, 25(1): 13-24. 
6. Weiss J. 1984. The sine-Gordon equations: Complete and partial integrability, Journal of Mathematical Physics, 25(7): 2226-35.

7. Weiss J. 1986. Bäcklund transformation and the Painlevé property, Journal of Mathematical Physics, 27(5): 1293-305.

8. Hone AN. 2009. Painlevé tests, singularity structure and integrability. Integrability: Springer; p. 245-77.

9. Hao X, Liu Y, Li Z, Ma W. 2019. Painlevé analysis, soliton solutions and lump-type solutions of the (3+ 1)-dimensional generalized KP equation, Journal of Computers Mathematics with Applications, 77(3): 724-30.

10. Wazwaz A. 2019. The integrable time-dependent sine-Gordon equation with multiple optical kink solutions, Optik, 128: 605-610.

11. Yee TL.2018; The Painlevé Test for a System of Coupled Equations from Nonlinear Birefringence, Journal of Applied Mathematical Sciences, 12(1): 27-36.

12. Singh M, Gupta R.2019; Group classification, conservation laws and Painlevé analysis for KleinGordon-Zakharov equations in (3+1)-dimension, Pramana - Journal Physics, 92(1): 1.

13. Jimbo M, Kruskal M, Miwa T.1982; Painlevé test for the self-dual Yang-Mills equation, Journal Physics Letters A, 92(2): 59-60.

14. Conte R, Fordy AP, Pickering A.1993; A perturbative Painlevé approach to nonlinear differential equations, Journal Physica D: Nonlinear Phenomena, 69(1-2): 33-58.

15. Fordy A, Pickering A.1991; Analysing negative resonances in the Painlevé test, Journal Physics Letters A, 160(4): 347-54. 